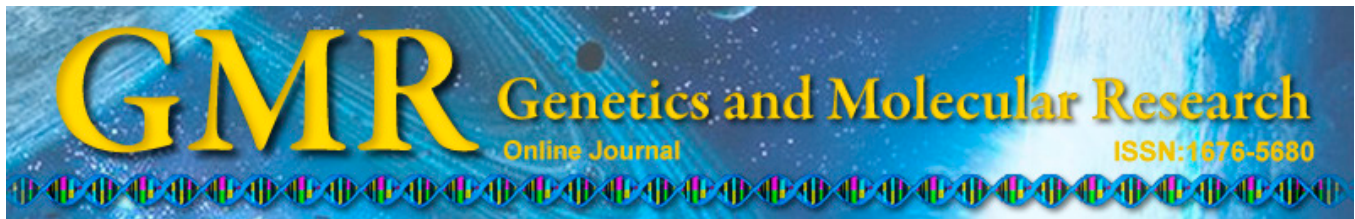

\title{
Correlation between p53 and epidermal growth factor receptor expression in breast cancer classification
}

\author{
X.Z. Wang, Q. Liu, J.J. Sun, W.S. Zuo, D.W. Hu, S.G. Ma, D.B. Mu and \\ Z.Y.Yu \\ Breast Disease Center \& Basic Research Center of Shandong Cancer Hospital, \\ School of Medicine and Life Sciences, University of Jinan, \\ Shandong Academy of Medical Sciences, Jinan, China \\ Corresponding author: Z.Y. Yu \\ E-mail: zhiyongyucn@126.com
}

Genet. Mol. Res. 14 (2): 4282-4290 (2015)

Received June 27, 2014

Accepted October 6, 2014

Published April 28, 2015

DOI http://dx.doi.org/10.4238/2015.April.28.10

\begin{abstract}
This study aimed to explore new opportunities for developing targeted therapy for triple-negative breast cancer (TNBC) by analyzing the significance and association between p53 and epidermal growth factor receptor (EGFR) expression in different molecular subtypes of breast cancer. The clinical and pathological data of 264 patients with breast cancer receiving surgery in our hospital from January 2012 to August 2013 were retrospectively analyzed. According to the expression of estrogen receptor, progesterone receptor, human epidermal growth factor receptor 2 (HER2), Ki-67, CK5/6, p53, and EGFR detected by immunohistochemical methods, breast cancer was divided into four molecular subtypes. Then, the expression of p53 and EGFR as well as their correlation in the different subtypes were determined. Among the four subtypes, luminal B breast cancer was the most common type. TNBC and HER2-enriched breast cancer had larger tumor sizes with higher expression of Ki-67 as compared with the luminal types. TNBC had a lower lymph node metastasis rate but
\end{abstract}


higher CK5/6 and EGFR expression than the other three types. The expression of p53 was higher in luminal B, HER2-enriched, and triplenegative breast cancers, and this was positively correlated with the expression of EGFR in TNBC but not in the other subtypes. p53 and EGFR expression was positively correlated in TNBC, which enables us to explore the molecular biological characteristics of TNBC, so as to provide new ideas for the treatment of TNBC.

Key words: Breast neoplasm; Molecular subtypes; p53; EGFR; Immunohistochemistry

\section{INTRODUCTION}

Breast cancer is the most common malignancy in women, as well as the main reason for female cancer-related deaths. With the advances in molecular biological research, a more in-depth understanding of breast cancer has been developed. Nowadays, breast cancer can be generally classified into luminal A, luminal B, human epidermal growth factor receptor 2 (HER2)-enriched, and triple negative breast cancer (TNBC) based on the presence or absence of the estrogen receptor (ER), progesterone receptor (PR), HER2, and Ki-67 (Ihemelandu et al., 2007; Muñoz et al., 2009; Wiechmann et al., 2009; Park et al., 2012). Breast cancer is a heterogeneous disease consisting of different molecular subtypes with different molecular biological characteristics and clinical behaviors. Sometimes, different molecular biological characteristics and different outcomes can even be observed in one molecular subtype of breast cancer (Raica et al., 2009). Luminal A breast cancer tends to have a better outcome, while TNBC has a strong aggressiveness, fast disease progression, easier metastasis and recurrence, poor prognosis, and other negative clinical features (Carey et al., 2006; Lin et al., 2008; Dawood et al., 2009). To date, there is no effective targeted therapy for TNBC. Because these tumors are not sensitive to endocrine therapy, chemotherapy is the main treatment for TNBC. Therefore, it is essential to determine additional reliable molecular markers to further characterize the molecular biological features of breast cancer, so as to better determine patient prognosis and guide their individual treatment.

p53 is a tumor suppressor gene that can induce apoptosis after DNA damage (Sjögren et al., 1996). p53 mutations play an important role in tumorigenesis. It was reported that p53 is not highly expressed in breast cancer (Sjögren et al., 1996), but it has been found to be highly expressed in TNBC (Grob et al., 2012). Epidermal growth factor receptor (EGFR) can promote tumor cell migration and increase the invasiveness of tumor cells. The EGFR positivity rate is about $57 \%$ in TNBC but only $8 \%$ in non-TNBC (Sparano et al., 2009). Studies have shown that mutations in the $p 53$ gene can lead to loss of function of the $p 63$ gene and thereby cause EGFR gene amplification. This ultimately promotes the formation of TNBC (Shapira et al., 2013). Both p53 and EGFR may be involved in the formation of TNBC, resulting in decreased effectiveness for breast cancer treatments that target EGFR alone (Masuda et al., 2012). Recent studies have shown that EGFR inhibitor has a lethal effect on lung cancer cells expressing wild-type p53 and mutated EGFR, but it is tolerated by the lung cancer cells expressing both p53 and EGFR mutations (Huang et al., 2011), indicating that there may be some association between $\mathrm{p} 53$ and EGFR expression in TNBC, which may assist in the deter- 
mination of therapeutic targets for TNBC. In this study, we retrospectively analyzed the clinical and pathological data of 264 patients with operable breast cancer and their immunohistochemical results, so as to explore the correlation between p53 and EGFR expression in the different molecular subtypes of breast cancer. Our results would provide further understanding of the molecular biological behavior of breast cancer and broaden the treatment strategies for breast cancer, particularly for TNBC.

\section{SUBJECTS AND METHODS}

\section{Clinical data of the subjects}

We retrospectively analyzed the clinical and pathological data of 264 patients with invasive breast cancer collected from the Pathology Department of Shandong Cancer Hospital. All patients underwent surgery at the Breast Disease Center of Shandong Cancer Hospital between January 2012 and August 2013. Patients receiving neoadjuvant therapy or having unknown $\mathrm{T}$ stages incised at a different hospital prior to surgery were excluded from the study. All of the pathological and immunohistochemical slices were reviewed by two experienced pathologists. The immunohistochemical indicators included ER, PR, HER2, CK5/6, Ki-67, p53, and EGFR. Clinical data such as age of onset, age of menarche, menopausal status, tumor size, lymph node metastasis, pathological staging, and other indicators were also reviewed. The breast cancer was then classified based on the cancer staging of American Joint Committee on Cancer (AJCC, 7th edition, 2010). This study was approved by the Ethics Committee of Shandong Cancer Hospital. Clinical data involved in this study were used with the consent of the patients themselves.

\section{Interpretation of immunohistochemical results and the defined criteria for molecular typing}

The streptavidin-peroxidase immunohistochemical method was used in this study. The DAB kit was from Fuzhou Maxim Biotechnology Co., Ltd. (Fujian, China). The primary antibodies used in this study included ER and PR (Beijing ZSGB Biotechnology Co., Ltd., Beijing, China), HER2, p53, and EGFR (Dako, Carpinteria, CA, USA), as well as CK5/6 and Ki-67 (Maxim, Fujian, China). Tumor cells with nuclei positively stained by ER, PR, p53, and Ki-67 were interpreted as immunopositive, while the positive expression for HER2, EGFR, and CK5/6 was observed in the cellular membrane or cytoplasm (Figure 1). The immunopositive staining of the cells was determined according to their proportion among the total cells. ER- or PR-positive tumors were determined by at least $1 \%$ of nuclei positively stained (Hammond et al., 2010), while $20 \%$ of nuclei positively stained by Ki-67 could be regarded as high expression (Goldhirsch et al., 2013). According to the recommendations from the American Society of Clinical Oncology and College of American Pathologists, HER2 expression can be classified as HER2-positive (score 3+), suspected HER2-positive (score 2+) and HER2-negative (score 0 or 1+). For those with suspected HER2-positive tumors, fluorescence in situ hybridization should be used to confirm its expression. For p53, EGFR, and CK5/6, at least 10\% of cells positively stained were considered as positive. According to the Expert Consensus of the 2013 St. Gallen International Breast Cancer Conference (Goldhirsch et al., 2013), breast cancer can be divided into four subtypes on the basis of their molecular markers (Table 1). 


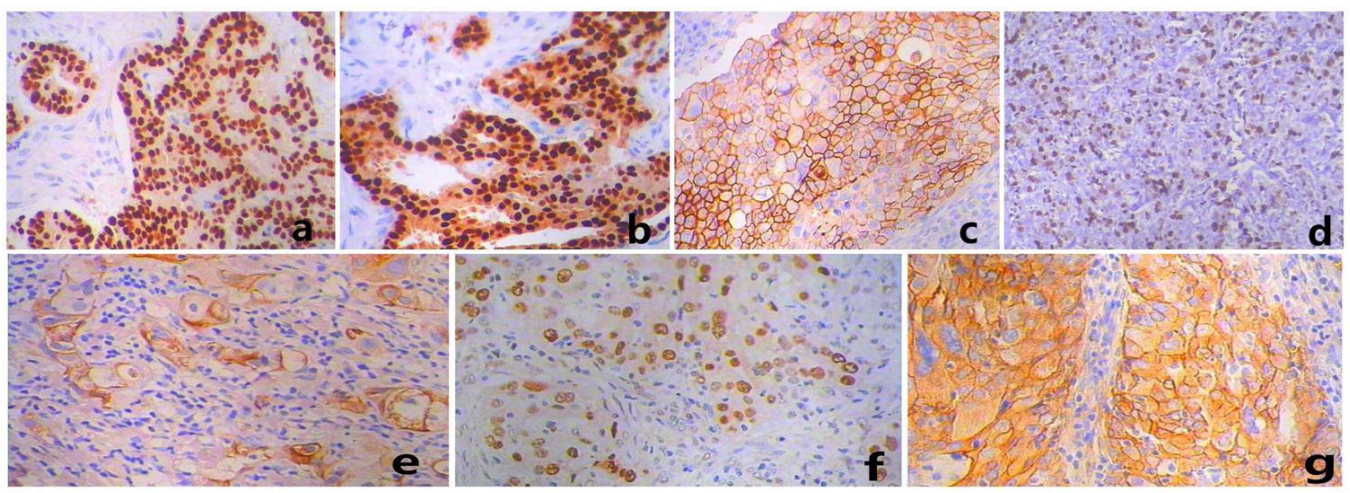

Figure 1. Expression of ER, PR, HER2, p53, EGFR, CK5/6, and Ki-67 in invasive breast cancer determined by immunohistochemistry (SP, 200X). a. ER-positive (70\%); b. PR-positive (60\%); c. HER2-positive (3+); d. Ki-67positive (50\%); e. CK5/6-positive; f. p53-positive; and g. EGFR-positive.

\begin{tabular}{l} 
Table 1. Breast cancer typing criteria. \\
\begin{tabular}{lllll} 
& & & \\
Subtypes & ER & HER2 & PR & Ki-67 \\
\hline Luminal A & Positive & Negative & $\geq 20 \%{ }^{\mathrm{a}}$ & Low expression \\
Luminal B & Positive & Negative & $<20 \%^{\mathrm{a}}$ or high Ki-67 expression & \\
HER2- & Positive & Positive & & \\
HER2 + & Negative & Positive & Negative & \\
HER2 overexpression & Negative & Negative & Negative & \\
TNBC & & &
\end{tabular} \\
\hline
\end{tabular}

aPrat et al. (2013).

\section{Statistical analysis}

Data were analyzed using the SPSS 17.0 software (SPSS Inc., Chicago, IL, USA). Owing to the non-normal distribution of our enumeration data, the chi-square test was used to compare the differences in clinicopathological features between the different molecular subtypes of breast cancer. Spearman correlation analysis was used to compare the correlation of 553 expression with EGFR expression in the different molecular subtypes of breast cancer. The results were considered to be statistically significant at $\mathrm{P}<0.05$.

\section{RESULTS}

\section{Clinical and pathological features}

The ages of the 264 patients ranged from 25 to 78 years (mean, $48.3 \pm 10.8$ years; median, 49 years). Their clinical and pathological characteristics are shown in Table 2. The luminal B subtype of breast cancer accounted for the highest proportion $(37.5 \%, 99 / 264)$ of the 264 cases of breast cancer, among which the HER2-negative type accounted for $31.4 \%$ (83/264) and the HER2-positive type accounted for 6.1\% (16/264). The second most common subtype was TNBC, accounting for $26.1 \%$ (69/264), followed by the luminal A subtype $(23.9 \%, 63 / 264)$ and the HER2-enriched subtype (12.5\%, 33/264) (Table 3). 
Table 2. Overview of the clinical and pathological features of breast cancer.

\begin{tabular}{|c|c|c|}
\hline Variables & No. of cases & Proportion $(\%)$ \\
\hline \multicolumn{3}{|l|}{ Age of onset } \\
\hline$<49$ years & 149 & 56.4 \\
\hline$\geq 49$ years & 115 & 43.6 \\
\hline \multicolumn{3}{|l|}{ Age of menarche } \\
\hline$<15$ years & 106 & 40.2 \\
\hline$\geq 15$ years & 158 & 59.8 \\
\hline \multicolumn{3}{|l|}{ Menopausal status } \\
\hline Yes & 92 & 34.8 \\
\hline No & 172 & 65.2 \\
\hline \multicolumn{3}{|l|}{ Tumor size } \\
\hline$\leq \mathrm{T}_{1}(\leq 2 \mathrm{~cm})$ & 131 & 49.6 \\
\hline$>\mathrm{T}_{1}^{1}(>2 \mathrm{~cm})$ & 133 & 50.4 \\
\hline \multicolumn{3}{|c|}{ Lymph node metastasis } \\
\hline Positive & 129 & 48.9 \\
\hline Negative & 135 & 51.1 \\
\hline \multicolumn{3}{|l|}{ Pathological staging } \\
\hline I & 78 & 29.5 \\
\hline II & 136 & 51.5 \\
\hline III & 50 & 19.0 \\
\hline ER+ & 163 & 61.6 \\
\hline $\mathrm{PR}+$ & 116 & 43.9 \\
\hline HER2+ & 50 & 18.9 \\
\hline $\mathrm{Ki}-67+(<20 \%)$ & 98 & 37.1 \\
\hline $\mathrm{Ki}-67+(\geq 20 \%)$ & 166 & 62.9 \\
\hline CK5/6+ & 50 & 18.9 \\
\hline p53+ & 88 & 33.4 \\
\hline EGFR+ & 39 & 14.8 \\
\hline
\end{tabular}

Table 3. Relationship between the molecular subtypes of breast cancer and the clinicopathological features.

\begin{tabular}{|c|c|c|c|c|c|c|c|}
\hline \multirow[t]{2}{*}{ Variables } & \multirow[t]{2}{*}{ Luminal A } & \multicolumn{2}{|c|}{ Luminal B } & \multirow[t]{2}{*}{ HER2-enriched } & \multirow[t]{2}{*}{ TNBC } & \multirow[t]{2}{*}{$\chi^{2}$} & \multirow[t]{2}{*}{$\mathrm{P}$ value } \\
\hline & & HER2 (-) & $\operatorname{HER} 2(+)$ & & & & \\
\hline Total cases & $63(23.9 \%)$ & $83(31.4 \%)$ & $16(6.1 \%)$ & $33(12.5 \%)$ & $69(26.1 \%)$ & & \\
\hline \multicolumn{8}{|l|}{ Age of onset } \\
\hline$<49$ years & 38 & 49 & 11 & 14 & 37 & 4.458 & 0.348 \\
\hline$\geq 49$ years & 25 & 34 & 5 & 19 & 32 & & \\
\hline \multicolumn{8}{|c|}{ Age of menarche } \\
\hline$<15$ years & 28 & 32 & 9 & 12 & 25 & 2.935 & 0.569 \\
\hline$\geq 15$ years & 35 & 51 & 7 & 21 & 44 & & \\
\hline \multicolumn{8}{|c|}{ Menopausal status } \\
\hline Yes & 20 & 31 & 0 & 11 & 30 & 11.351 & 0.023 \\
\hline No & 43 & 52 & 16 & 22 & 39 & & \\
\hline \multicolumn{8}{|l|}{ Tumor sizes } \\
\hline$\leq 2 \mathrm{~cm}$ & 39 & 43 & 10 & 9 & 30 & 12.658 & 0.013 \\
\hline$>2 \mathrm{~cm}$ & 24 & 40 & 6 & 24 & 39 & & \\
\hline \multicolumn{8}{|c|}{ Lymph node metastasis } \\
\hline Positive & 33 & 49 & 8 & 16 & 23 & 10.420 & 0.034 \\
\hline Negative & 30 & 34 & 8 & 17 & 46 & & \\
\hline \multicolumn{8}{|c|}{ Pathological staging } \\
\hline I & 24 & 20 & 7 & 4 & 23 & 15.559 & 0.049 \\
\hline II & 34 & 42 & 7 & 20 & 33 & & \\
\hline III & 5 & 21 & 2 & 9 & 13 & & \\
\hline \multicolumn{8}{|l|}{ Ki-67 } \\
\hline$<20 \%$ & 63 & 18 & 4 & 4 & 11 & 137.111 & 0.001 \\
\hline$\geq 20 \%$ & 0 & 65 & 12 & 29 & 58 & & \\
\hline \multicolumn{8}{|l|}{$\mathrm{CK} 5 / 6$} \\
\hline Positive & 1 & 8 & 0 & 5 & 36 & 70.722 & 0.001 \\
\hline Negative & 62 & 75 & 16 & 28 & 33 & & \\
\hline \multicolumn{8}{|l|}{ p53 } \\
\hline Positive & 6 & 29 & 8 & 15 & 30 & 23.545 & 0.001 \\
\hline Negative & 57 & 54 & 8 & 18 & 39 & & \\
\hline \multicolumn{8}{|l|}{ EGFR } \\
\hline Positive & 1 & 3 & 1 & 5 & 29 & 58.548 & 0.001 \\
\hline Negative & 62 & 80 & 15 & 28 & 40 & & \\
\hline
\end{tabular}


The molecular subtypes of breast cancer were not associated with age of onset or age of menarche ( $\mathrm{P}>0.05$, both). However, the luminal B (HER2-positive) subtype was more common in premenopausal women, showing a significant difference from the other subtypes $(\mathrm{P}=0.023)$. The tumor sizes in the TNBC and HER2-enriched subtypes were larger than those in the other two subtypes $(\mathrm{P}=0.013)$ with a higher expression of Ki-67 $(\mathrm{P}=0.001)$. TNBC had the lowest positive rate of lymph node metastasis $(\mathrm{P}=0.034)$ but higher positive rates of CK5/6 and EGFR ( $\mathrm{P}=0.0001$, both). In the HER2-enriched subtype, the proportion of pathological stage III cases was remarkably higher than among the other three subtypes $(\mathrm{P}=0.049)$. p53 expression was high in the luminal B, HER2-enriched, and TNBC subtypes but low in the luminal A subtype (Table 3).

\section{Correlation between p53 and EGFR expression in different molecular subtypes of breast cancer}

The expression of p53 and EGFR was $43.5 \%(30 / 69)$ and $42.0 \%(29 / 69)$ in TNBC, 9.5\% (6/63) and 1.6\% (1/63) in the luminal A subtype, 34.9\% (29/83) and 3.6\% (3/83) in the luminal B subtype with HER2-negative, 50\% (8/16) and 6.25\% (1/16) in luminal B with HER2-positive, $45.5 \%(15 / 33)$ and $15.2 \%(5 / 33)$ in the HER2-enriched subtype and $43.5 \%$ $(30 / 69)$ and $42.0 \%(29 / 69)$ in TNBC, respectively. Spearman correlation analysis showed that p53 expression was positively correlated with EGFR expression in invasive breast cancer $(r=$ $0.226, \mathrm{P}=0.001$; Table 4). Further analysis showed that the correlation of $\mathrm{p} 53$ expression with EGFR expression only existed in TNBC $(r=0.319, \mathrm{P}=0.007$; Table 5$)$ but not in the other molecular subtypes of breast cancer $(\mathrm{P}>0.05$; Table 5$)$.

\begin{tabular}{|c|c|c|c|c|}
\hline \multirow[t]{2}{*}{ p53 expression } & \multicolumn{2}{|c|}{ EGFR expression } & \multirow[t]{2}{*}{$\mathrm{r}$} & \multirow[t]{2}{*}{$P$ value } \\
\hline & Positive & $\overline{\text { Negative }}$ & & \\
\hline Positive & 23 & 65 & 0.226 & 0.001 \\
\hline Negative & 16 & 160 & & \\
\hline
\end{tabular}

\begin{tabular}{|c|c|c|c|c|c|}
\hline \multirow[t]{2}{*}{ p53/EGFR } & \multirow[t]{2}{*}{ Luminal A } & \multicolumn{2}{|c|}{ Luminal B } & \multirow[t]{2}{*}{ HER2-enriched } & \multirow[t]{2}{*}{ TNBC } \\
\hline & & HER2 (-) & HER2 (+) & & \\
\hline +/+ & 0 & 2 & 1 & 2 & 18 \\
\hline$+/-$ & 6 & 27 & 7 & 13 & 12 \\
\hline$-/+$ & 1 & 1 & 0 & 3 & 11 \\
\hline$-/-$ & 56 & 53 & 8 & 15 & 28 \\
\hline 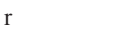 & -0.041 & 0.129 & 0.258 & -0.046 & 0.319 \\
\hline$P$ value & 0.748 & 0.246 & 0.334 & 0.798 & 0.007 \\
\hline
\end{tabular}

\section{DISCUSSION}

The $p 53$ gene plays an important role in the regulation of cell cycles. The wild-type p53 gene is involved in cell cycle regulation, preventing the transition from the G1 phase to the $\mathrm{S}$ phase. Thus, it is a negative regulator for cell division and proliferation. However, when 
it is mutated, it can no longer arrest cell proliferation or induce apoptosis, but instead leads to uncontrolled cell growth and tumor formation. Our findings showed that the overall expression of p53 was low in invasive breast cancer (33.4\%), with $37.4 \%$ positivity in the luminal B subtype, $43.5 \%$ in TNBC, and $45.5 \%$ in the HER2-enriched subtype. Curtis et al. (2012) analyzed the $p 53$ gene in 2000 cases of breast cancer specimens and found that the mutation rate of $p 53$ in the luminal A subtype was 5\%, while it was $13 \%$ in luminal B, 34\% in TNBC, and $22 \%$ in the HER2-enriched subtype. It has been demonstrated that overexpression of EGFR in breast tumors is associated with larger tumor size, poor differentiation, and poor prognosis. EGFR overexpression can be seen in each of the molecular subtypes of breast cancer, but it is found at a higher rate in TNBC and inflammatory breast cancer (Burness et al., 2010; Masuda et al., 2012). In this study, the positive rate of EGFR was $42 \%$ in TNBC, slightly lower than that reported by Dent et al. (2007). Other subtypes of breast cancer showed lower EGFR expression, which was $1.6 \%$ in luminal A, $4.0 \%$ in luminal B, and $15.2 \%$ in the HER2-enriched subtype. We found that p53 expression was positively correlated with EGFR expression in invasive breast cancer, and a further analysis found that the correlation existed only in TNBC but not in the other molecular subtypes. It was reported that mutation in the $p 53$ gene leads to $p 63$ gene mutation and loss of function, which would cause EGFR gene amplification and ultimately contribute to the formation of TNBC (Shapira et al., 2013). EGFR inhibitors exert their lethal effect on lung cancer cells expressing wild-type $p 53$ gene and mutated $E G F R$ gene but they have no effect on the lung cancer cells expressing both mutated $p 53$ and mutated $E G F R$ gene (Huang et al., 2011), suggesting that p53 may have a regulatory effect on EGFR inhibitors. The low expression of $\mathrm{p} 53$ in breast cancer overall, but its high expression in TNBC suggests that we need to launch a new understanding of the EGFR signaling pathway by targeting p53 expression, so as to explore a new therapeutic approach for TNBC. Reconstruction of the wild-type $p 53$ gene may overcome the resistance to EGFR inhibitors in patients with TNBC, and $p 53$ gene therapy combined with targeted inhibition of EGFR may change the treatment model for some patients with TNBC.

In this study, the Expert Consensus of the 2013 St. Gallen International Breast Cancer Conference was used to analyze the subtypes of breast cancer and showed that luminal B was the most common subtype (37.5\%) in this group of subjects, followed by TNBC (26.1\%), luminal A (23.9\%), and the HER2-enriched subtype (12.5\%), findings consistent with the results from El Fatemi et al. (2012), Howland et al. (2013), and Goldhirsch et al. (2013). In this study, the positive rate of CK5/6 was $52.2 \%$ in TNBC. CK5/6 is a basal keratin expressed in the basal lamina of epithelial tissues, which may be a marker of basal-like breast cancer (BLBC). Thus, CK5/6-positive TNBC is more likely to be BLBC. There is an overlap of about $80 \%$ of BLBC with TNBC, thus CK5/6 expression can be used as one marker for BLBC. HER2-enriched breast cancer and TNBC are more malignant, more aggressive and grow more rapidly. In this study, we also found that the tumor sizes were larger in HER2-enriched breast cancer and TNBC, which was also reported by Park et al. (2012). Currently, HER2-targeted therapies have offered more benefits for patients with HER2-enriched breast cancer, but there is still no effective targeted therapy for patients with TNBC. Thus, it is necessary to develop a new treatment modality for TNBC. This study also determined that the lymph node metastasis rate was higher in luminal B with HER2-negative (59.0\%) but lower in TNBC (33.3\%). Bennis et al. (2012) also found that the luminal B subtype of breast cancer is prone to lymph node metastasis $(74 \%)$, while TNBC appears to have a lower incidence of lymph node metastasis $(55.2 \%)$, suggesting that the luminal B subtype of breast cancer is more likely to metastasize via lymph 
nodes, but TNBC may be more likely to metastasize via blood circulation.

No molecular subtype of breast cancer was associated with the age of onset in this study, but the luminal B (HER2-positive) subtype was more common in premenopausal women, while the other molecular subtypes displayed no correlation with menopausal status $(\mathrm{P}>0.05)$. Li et al. (2013) also reported that molecular subtypes of breast cancer were not correlated with age of onset or menopausal status, while TNBC is more common in patients below 40 years in California, U.S. (Bauer et al., 2007). In addition, researchs from the Carolina Breast Cancer Study showed that TNBC more commonly occurs in premenopausal women in North Carolina (Carey et al., 2006; O'Brien et al., 2010). The disparities between these studies may result from the racial differences in the samples. Furthermore, it is possible that the fact that all samples were from a single center in this study may also contribute to the differences. Thus, a larger sample size from multiple centers will be included in future studies to verify the results of this study.

In conclusion, different molecular subtypes of breast cancer demonstrated different clinical and pathological features. The expressions of p53 and EGFR were higher and positively correlated with each other in TNBC, while in the other subtypes, their expressions were lower and had no correlation with each other. Gene therapy to reconstruct wild-type $p 53$ combined with therapy targeting the inhibition of EGFR may bring new opportunities for patients with TNBC.

\section{ACKNOWLEDGMENTS}

Research supported by grants from the Natural Science Foundation of Shandong Province (\#ZR2012HL34).

\section{REFERENCES}

Bauer KR, Brown M, Cress RD, Parise CA, et al. (2007). Descriptive analysis of estrogen receptor (ER)-negative, progesterone receptor (PR)-negative, and HER2-negative invasive breast cancer, the so-called triple-negative phenotype: a population-based study from the California cancer Registry. Cancer 109: 1721-1728.

Bennis S, Abbass F, Akasbi Y, Znati K, et al. (2012). Prevalence of molecular subtypes and prognosis of invasive breast cancer in north-east of Morocco: retrospective study. BMC Res. Notes 5: 436-440.

Burness ML, Grushko TA and Olopade OI (2010). Epidermal growth factor receptor in triple-negative and basal-like breast cancer: promising clinical target or only a marker? Cancer J. 16: 23-32.

Carey LA, Perou CM, Livasy CA, Dressler LG, et al. (2006). Race, breast cancer subtypes, and survival in the Carolina Breast Cancer Study. JAMA 295: 2492-2502.

Curtis C, Shah SP, Chin SF, Turashvili G, et al. (2012). The genomic and transcriptomic architecture of 2,000 breast tumours reveals novel subgroups. Nature 486: 346-352.

Dawood S, Broglio K, Esteva FJ, Yang W, et al. (2009). Survival among women with triple receptor-negative breast cancer and brain metastases. Ann. Oncol. 20: 621-627.

Dent R, Trudeau M, Pritchard KI, Hanna WM, et al. (2007). Triple-negative breast cancer: clinical features and patterns of recurrence. Clin. Cancer Res. 13: 4429-4434.

El Fatemi H, Chahbouni S, Jayi S, Moumna K, et al. (2012). Luminal B tumors are the most frequent molecular subtype in breast cancer of North African women: an immunohistochemical profile study from Morocco. Diagn. Pathol. 7: 170.

Goldhirsch A, Winer EP, Coates AS, Gelber RD, et al. (2013). Personalizing the treatment of women with early breast cancer: highlights of the St. Gallen International Expert Consensus on the Primary Therapy of Early Breast Cancer 2013. Ann. Oncol. 24: 2206-2223.

Grob TJ, Heilenkötter U, Geist S, Paluchowski P, et al. (2012). Rare oncogenic mutations of predictive markers for targeted therapy in triple-negative breast cancer. Breast Cancer Res. Treat. 134: 561-567.

Hammond ME, Hayes DF, Dowsett M, Allred DC, et al. (2010). American Society of Clinical Oncology/College of American Pathologists guideline recommendations for immunohistochemical testing of estrogen and progesterone 
receptors in breast cancer (unabridged version). Arch. Pathol. Lab. Med. 134: e48-e72.

Howland NK, Driver TD, Sedark MP, Wen X, et al. (2013). Lymph node involvement in immunohistochemistry-based molecular classifications of breast cancer. J. Surg. Res. 185: 697-703.

Huang S, Benavente S, Armstrong EA, Li C, et al. (2011). p53 modulates acquired resistance to EGFR inhibitors and radiation. Cancer Res. 71: 7071-7079.

Ihemelandu CU, Leffall LD Jr, Dewitty RL, Naab TJ, et al. (2007). Molecular breast cancer subtypes in premenopausal and postmenopausal African-American women: age-specific prevalence and survival. J. Surg. Res. 143: 109-118.

Li J, Jia S, Zhang W, Zhang Y, et al. (2013). Survival analysis based on clinicopathological data from a single institution: chemotherapy intensity would be enhanced in patients with positive hormone receptors and positive HER2 in China who cannot afford the target therapy. ISRN Oncol. 2013: 606389.

Lin NU, Claus E, Sohl J, Razzak AR, et al. (2008). Sites of distant recurrence and clinical outcomes in patients with metastatic triple-negative breast cancer: high incidence of central nervous system metastases. Cancer 113: 2638-2645.

Masuda H, Zhang D, Bartholomeusz C, Doihara H, et al. (2012). Role of epidermal growth factor receptor in breast cancer. Breast Cancer Res. Treat. 136: 331-345.

Muñoz M, Fernández-Aceñero MJ, Martín S and Schneider J (2009). Prognostic significance of molecular classification of breast invasive ductal carcinoma. Arch. Gynecol. Obstet. 280: 43-48.

O'Brien KM, Cole SR, Tse CK, Perou CM, et al. (2010). Intrinsic breast tumor subtypes, race, and long-term survival in the Carolina Breast Cancer Study. Clin. Cancer Res. 16: 6100-6110.

Park S, Koo JS, Kim MS, Park HS, et al. (2012). Characteristics and outcomes according to molecular subtypes of breast cancer as classified by a panel of four biomarkers using immunohistochemistry. Breast 21: 50-57.

Prat A, Cheang MC, Martin M, Parker JS, et al. (2013). Prognostic significance of progesterone receptor-positive tumor cells within immunohistochemically defined luminal A breast cancer. J. Clin. Oncol. 31: 203-209.

Raica M, Jung I, Cimpean AM, Suciu C, et al. (2009). From conventional pathologic diagnosis to the molecular classification of breast carcinoma: are we ready for the change. Rom. J. Morphol. Embryol. 50: 5-13.

Shapira I, Lee A, Vora R and Budman DR (2013). P53 mutations in triple negative breast cancer upregulate endosomal recycling of epidermal growth factor receptor (EGFR) increasing its oncogenic potency. Crit. Rev. Oncol. Hematol. 88: 284-292.

Sjögren S, Inganäs M, Norberg T, Lindgren A, et al. (1996). The p53 gene in breast cancer: prognostic value of complementary DNA sequencing versus immunohistochemistry. J. Natl. Cancer Inst. 88: 173-182.

Sparano JA, Goldestin LJ, Childs BH, Shak S, et al. (2009). Genotypic characterization of phenotypically defined triplenegative breast cancer. J. Clin. Oncol. 27 (Suppl): 15s.

Wiechmann L, Sampson M, Stempel M, Jacks LM, et al. (2009). Presenting features of breast cancer differ by molecular subtype. Ann. Surg. Oncol. 16: 2705-2710. 